\title{
Mechanism of action of brexpiprazole: comparison with aripiprazole
}

\author{
Stephen M. Stahl \\ ISSUE: \\ Brexpiprazole is a new therapeutic agent that was recently approved for the \\ treatment of schizophrenia and for the adjunctive treatment of major depressive \\ disorder. Brexpiprazole has features that both overlap and contrast with a related \\ molecule, aripiprazole, and these features are discussed here.
}

\section{Take-Home Points}

- Brexpiprazole, like aripiprazole, is a dopamine multifunctional agent. Both have dopamine and serotonin receptor partial agonism as their pharmacological mechanism of action. Both are also known as "atypical antipsychotics" and as "adjunctive antidepressants.

- No head-to-head studies compare the efficacy or the safety of brexpiprazole versus aripiprazole, so comparisons can only be made on the basis of their pharmacologic properties, or by comparing separate clinical studies of each drug versus placebo.

- Pharmacologically, brexpiprazole has less intrinsic activity (ie, is less of a partial agonist and more of an antagonist) at D2 receptors than aripiprazole. Brexpiprazole also has much more potent actions at 5HT2A, 5HT1A, and alpha $1 \mathrm{~B}$ receptors than aripiprazole.

- Clinical differentiation appears to be mainly improved tolerability (eg, potentially less akathisia and extrapyramidal symptoms with brexpiprazole than aripiprazole) but similar efficacy for the 2 compounds both in schizophrenia and as adjunctive treatments in major depression

A new dopamine D2 receptor partial agonist, brexpiprazole, has been approved both for the treatment of schizophrenia and as adjunctive treatment for major depressive disorder. Since brexpiprazole (Figure 1$)^{1}$ is chemically and pharmacologically related to aripiprazole
(Figure 2), a D2 partial agonist marketed for several years for these same indications, ${ }^{1}$ the question naturally arises, "What is the difference between aripiprazole and brexpiprazole?"

\section{Brexpiprazole Has Less Intrinsic Activity than Aripiprazole}

The dopamine agonist spectrum goes from "silent" antagonism on the far left of Figure 3, which is pure antagonism without any agonist activity, to "full" agonism on the far right of Figure 3, which is the maximum amount of stimulation of the D2 receptor. Almost all antipsychotics are silent antagonists and lie to the far left on the spectrum. Dopamine itself is a full agonist and lies to the far right on the spectrum.

Note that toward the left hand part of the spectrum lie several interesting compounds that have been tested as antipsychotics. Those furthest to the right (OPC 4392 and bifeprunox) seem to be too activating and to have too much of a dopamine "kick" - more precisely, too much intrinsic activity. ${ }^{1}$ These agents have therefore failed as effective antipsychotics. A bit less intrinsic activity, and you have aripiprazole (Figure 3). However, aripiprazole's clinical profile of activation, agitation, and akathisia in some patients suggests that it has too much intrinsic activity for them. Thus, the pharmacology of brexpiprazole purposely "dialed down" the intrinsic activity another notch (Figure 3 ) to theoretically generate receptor binding properties at the D2 receptor that would make it less activating and possibly a better overall 


\section{BRAINSTORMS-Clinical Neuroscience Update}

Figure 1. Pharmacologic properties of aripiprazole shown qualitatively as an icon above and quantitatively as a binding strip below. The binding strip is composed of a series of boxes for potency of binding of the drug at each individual

neurotransmitter receptor indicated. Boxes are placed in rank order of how potently aripiprazole binds to the receptors, with the most potent and largest boxes to the far left and the weakest binding and smallest boxes to the far right. The vertical line indicates binding at the D2 dopamine receptor, generally targeted for 60-80\% occupancy in the treatment of schizophrenia. Thus, boxes to the left of this generally indicate greater degrees of occupancy at these receptors at therapeutic doses; boxes to the right generally indicate lower degrees of occupancy at these receptors at therapeutic doses. Thus, almost all receptors have binding affinities lower than that for the D2 receptor with aripiprazole. This includes the 5HT1A, 5HT2A, and alpha 1B receptors, for example.

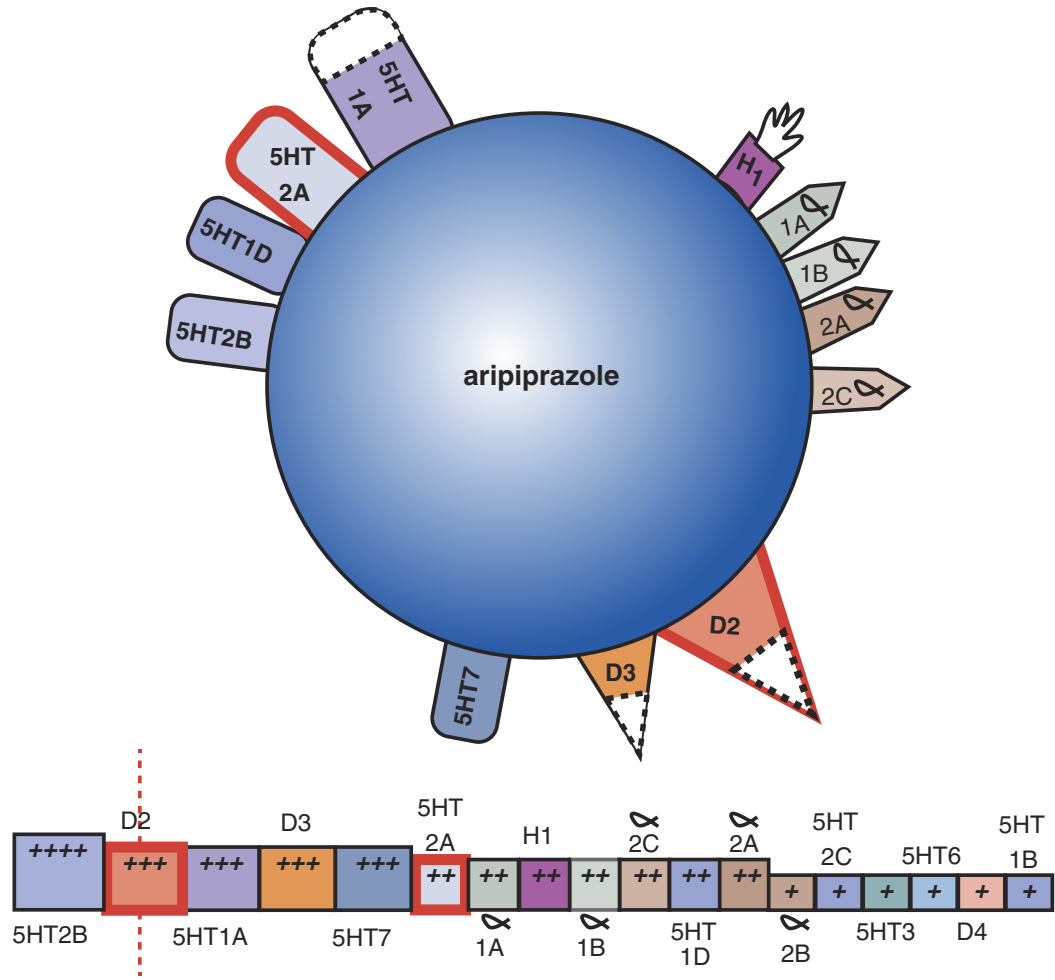

tolerability profile as an antipsychotic than aripiprazole. Whether brexpiprazole occupies the "sweet spot" on the agonist spectrum remains to be determined with headto-head trials with other antipsychotics, or with more clinical experience now that it is approved. However, brexpiprazole's preclinical and clinical profiles are so far consistent with its lesser amount of intrinsic activity on the agonist spectrum (Figure 3).

\section{Brexpiprazole Has More Potent Binding at 5HT2A, 5HT1A, and Alpha 1B Receptors Than Aripiprazole}

At least 3 receptor actions are known to mitigate the akathisia and extrapyramidal side effects (EPS) associated with blocking D2 dopamine receptors. These are
5HT2A antagonism, 5HT1A agonism, and alpha 1B antagonism. ${ }^{1-8}$ Each of these receptor actions has been discussed extensively elsewhere. ${ }^{1,9}$ Although aripiprazole does have actions at all 3 receptors, these actions are relatively weak (see Figures 1, and 4-6). That is, the binding strips for aripiprazole in these figures show boxes for potency of binding of the drug at individual neurotransmitter receptors. They are placed in rank order of how potently aripiprazole binds to the receptors, with the most potent and largest boxes to the far left and the weakest binding and smallest boxes to the far right. Comparing brexpiprazole with aripiprazole, we can see that brexpiprazole has much greater potency at each of these 3 receptors, ie, at the $5 \mathrm{HT} 2 \mathrm{~A}$ receptor (see the arrow of 5HT2A binding moving to the left in Figure 4), at the $5 \mathrm{HT} 1 \mathrm{~A}$ receptor (arrow of $5 \mathrm{HT} 1 \mathrm{~A}$ binding also moving to 


\section{BRAINSTORMS-Clinical Neuroscience Update}

Figure 2. Pharmacologic properties of brexpiprazole shown qualitatively as an icon above and quantitatively as a binding strip below. The binding strip is composed of a series of boxes for potency of binding of the drug at each individual

neurotransmitter receptor indicated. Boxes are placed in rank order of how potently brexpiprazole binds to the receptors, with the most potent and largest boxes to the far left and the weakest binding and smallest boxes to the far right. The vertical line indicates binding at the D2 dopamine receptor, generally targeted for 60-80\% occupancy in the treatment of schizophrenia. Thus, boxes to the left of this generally indicate greater degrees of occupancy at these receptors at therapeutic doses; boxes to the right generally indicate lower degrees of occupancy at these receptors at therapeutic doses. For brexpiprazole, in contrast to aripiprazole (Figure 1), the binding affinity at 5HT1A, 5HT2A, and alpha 1B receptors is equal to or higher than that for D2 receptors, indicating substantial occupancy of these 3 receptors at therapeutic doses.

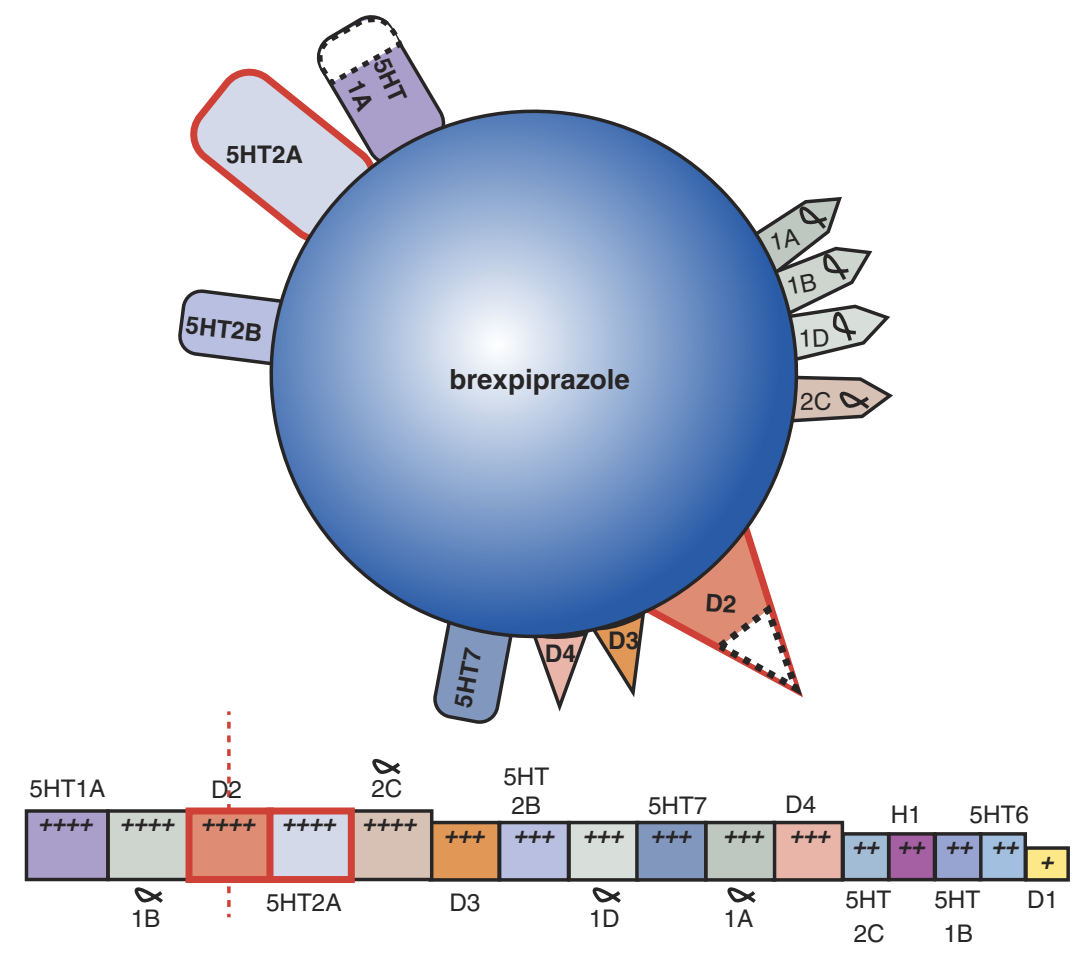

the left for brexpiprazole in Figure 5), and at the alpha 1B receptor (arrow of alpha $1 \mathrm{~B}$ binding moving to the left for brexpiprazole in Figure 6).

One additional difference between brexpiprazole and aripiprazole is that antihistamine properties (binding at the $\mathrm{H} 1$ receptor) is reduced for brexpiprazole compared to aripiprazole (arrow of $\mathrm{H} 1$ binding moving to the right for brexpiprazole in Figure 7). This suggests less potential for sedation, somnolence, and weight gain.

\section{Brexpiprazole May Be Less Activating with Less Akathisia or EPS than Aripiprazole}

Whereas the binding data comparing brexpiprazole and aripiprazole are robust and replicated, what this means in terms of functional activity in patients is not proven. However, the neuroscience of receptor binding and indications of how this is linked to functional actions in animal models can provide plausible hypotheses to be tested rigorously in patients-something that has not yet been done comparing brexpiprazole and aripiprazole. Numerous studies indicate that blocking 5HT2A or alpha $1 \mathrm{~B}$ receptors, or stimulating $5 \mathrm{HT} 1 \mathrm{~A}$ receptors can be associated with reduction of EPS, possibly due to enhanced release of downstream dopamine reversing some D2 blockade of receptors in the striatum. ${ }^{1-9}$ Empirically, agents with binding properties at these receptors more potent than actions at $\mathrm{D} 2$ receptors are those with the lowest EPS. ${ }^{1}$ Aripiprazole has actions at 5HT2A, 5HT1A, and alpha $1 \mathrm{~B}$ receptors that are less potent than its actions at D2 receptors, whereas 


\section{BRAINSTORMS-Clinical Neuroscience Update}

Figure 3. The dopamine agonist spectrum goes from "silent" antagonism on the far left, which is pure antagonism without any agonist activity, to "full" agonism on the far right, which is the maximum amount of stimulation of the D2 receptor. Almost all antipsychotics are silent antagonists and lie to the far left on the spectrum. Dopamine itself is a full agonist and lies to the far right on the spectrum. Novel antipsychotics lie between these two extremes, but closer to the antagonist end of the spectrum. OPC 4392 and bifeprunox seem to have too much of a dopamine "kick" - more precisely, too much intrinsic activity, or too far to the right on the spectrum - and have failed as effective antipsychotics. A bit less intrinsic activity, and you have aripiprazole. However, aripiprazole's clinical profile of activation, agitation, and akathisia in some patients suggests that it has too much intrinsic activity for them. Thus, the pharmacology of brexpiprazole purposely "dialed down" the intrinsic activity another notch, to theoretically generate receptor-binding properties at the D2 receptor that would make it less activating and possibly a better overall tolerability profile compared to aripiprazole.

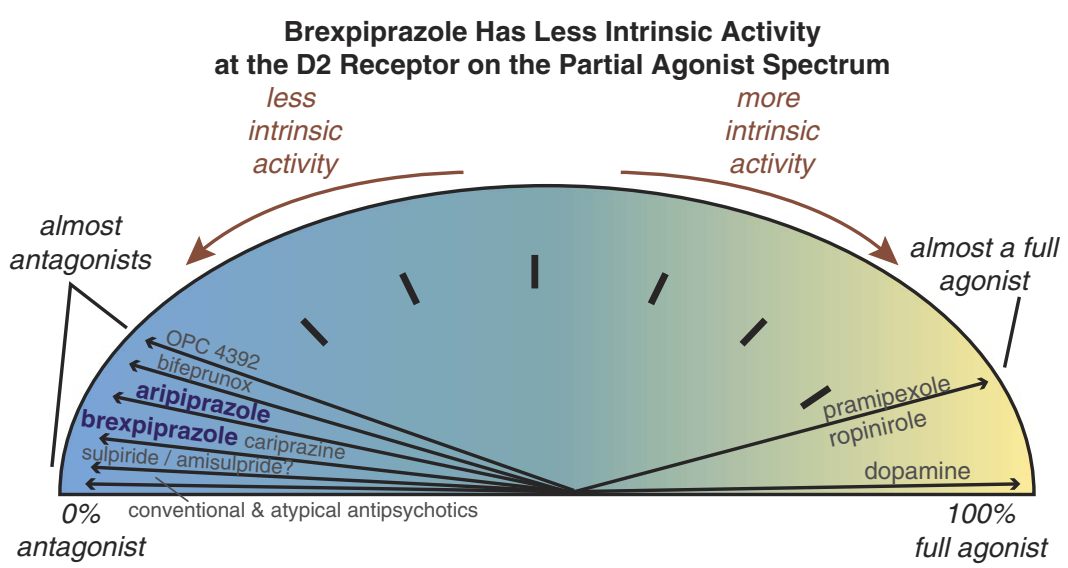

Figure 4. Comparing 5HT2A receptor binding of brexpiprazole versus aripiprazole. The binding strips for brexpiprazole and aripiprazole that appear separately in Figures 1 and 2 are shown together here. The D2 receptor affinity for both compounds is highlighted by a vertical dotted line. The 5HT2A receptor binding affinities for both compounds are labeled in this figure. The relative affinities of brexpiprazole and aripiprazole for the 5HT2A receptor are highlighted with a gray arrow moving to the left, indicating that brexpiprazole has substantially more potent $5 \mathrm{HT} 2 \mathrm{~A}$ receptor binding affinity than aripiprazole.

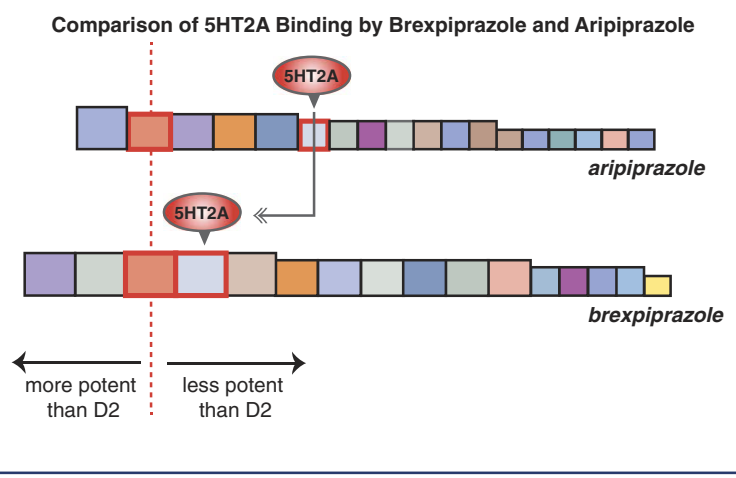

Figure 5. Comparing 5HT1A receptor binding of

brexpiprazole versus aripiprazole. The binding strips for brexpiprazole and aripiprazole that appear separately in Figures 1 and 2 are shown together here. The D2 receptor affinity for both compounds is highlighted by a vertical dotted line. The 5HT1A receptor binding affinities for both compounds are labeled in this figure. The relative affinities of brexpiprazole and aripiprazole for the 5HT1A receptor are highlighted with a gray arrow moving to the left, indicating that brexpiprazole has substantially more potent 5 HT1A receptor binding affinity than aripiprazole.

Comparison of 5HT1A Binding by Brexpiprazole and Aripiprazole

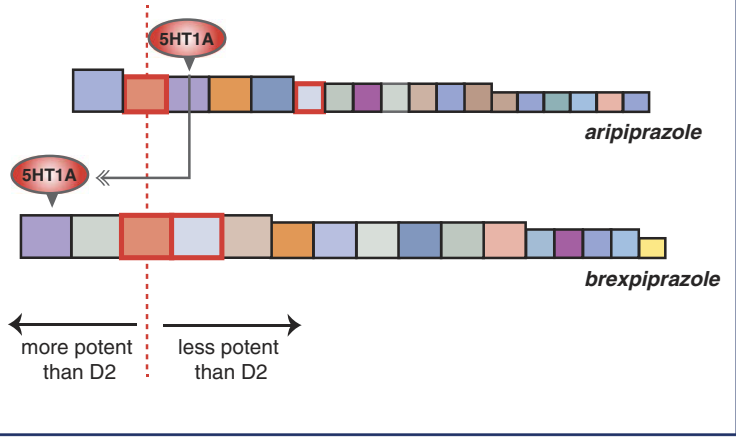




\section{BRAINSTORMS - Clinical Neuroscience Update}

Figure 6. Comparing alpha $1 \mathrm{~B}$ receptor binding of

brexpiprazole versus aripiprazole. The binding strips

for brexpiprazole and aripiprazole that appear separately

in Figures 1 and 2 are shown together here. The D2

receptor affinity for both compounds is highlighted

by a vertical dotted line. The alpha $1 \mathrm{~B}$ receptor binding

affinities for both compounds are labeled in this figure.

The relative affinities of brexpiprazole and aripiprazole

for the alpha 1B receptor are highlighted with a gray

arrow moving to the left, indicating that brexpiprazole

has substantially more potent alpha $1 \mathrm{~B}$ receptor binding affinity than aripiprazole.

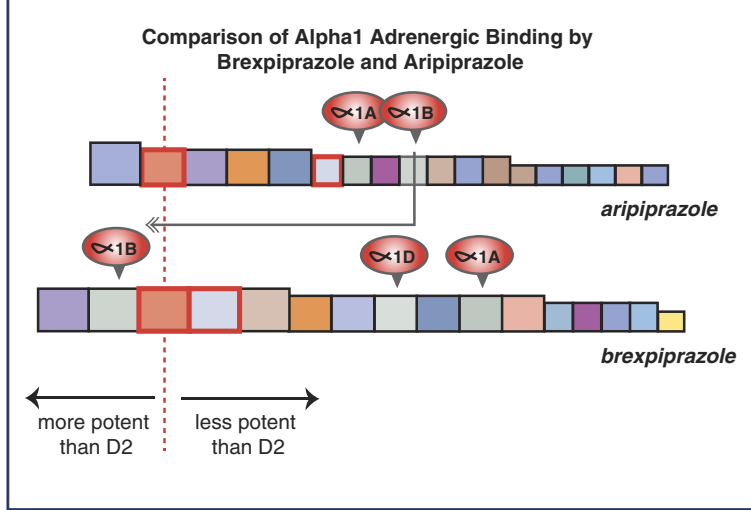

Figure 7. Comparing H1 histamine receptor binding of brexpiprazole versus aripiprazole. The binding strips for brexpiprazole and aripiprazole that appear separately in Figures 1 and 2 are shown together here. The D2 receptor affinity for both compounds is highlighted by a vertical dotted line. The alpha $1 \mathrm{~B}$ receptor binding affinities for both compounds are labeled in this figure. The relative affinities of brexpiprazole and aripiprazole for the alpha $1 \mathrm{~B}$ receptor are highlighted with a gray arrow moving to the right, indicating that brexpiprazole has substantially less potent alpha 1B receptor binding affinity than aripiprazole. $\mathrm{H} 1$ antagonism (ie, antihistaminic effects) is linked to somnolence, sedation, and weight gain.

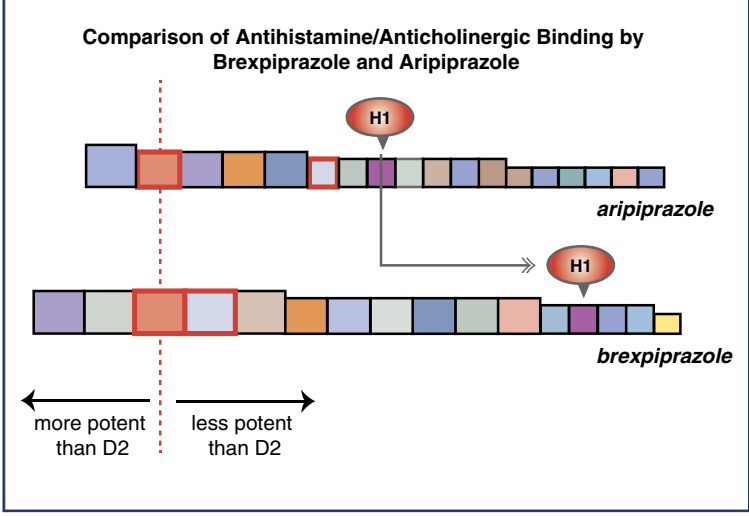

brexpiprazole has actions at these 3 receptors equally or more potent than at D2 receptors (see Figures 4-6). But, does brexpiprazole have less EPS or akathisia than aripiprazole?

Although the best way to address this question would be adequately powered head-to-head studies of brexpiprazole versus aripiprazole in patient populations, one preliminary way to explore whether brexpiprazole differs from aripiprazole is to look at drug-placebo differences across studies, such as those reviewed by Citrome ${ }^{10}$ and indicated in some well-organized tables. For example, looking at the percentage of patients with akathisia in the major depressive disorder studies where this side effect tends to be more problematic, aripiprazole had $25 \%$ akathisia compared to placebo with $4 \%$. Brexpiprazole had 9\% akathisia compared to placebo with $2 \%,{ }^{10}$ which is consistent with the predictions from the pharmacology that brexpiprazole should have less propensity for this side effect.

\section{Brexpiprazole Compared to Aripiprazole for Other Side Effects}

Looking at the number needed to harm (NNH) is a useful way to compare side effects of drugs, ${ }^{10}$ even in separate but similarly designed multicenter trials against placebo. For weight gain, the $\mathrm{NNH}$ was similar for brexpiprazole and aripiprazole in schizophrenia studies, but brexpiprazole had about twice the $\mathrm{NNH}$ for depression studies, ${ }^{10}$ meaning that brexpiprazole caused significant weight gain about half as often as aripiprazole did. For sedation, the NNH showed that brexpiprazole caused more sedation than aripiprazole in schizophrenia but less sedation than aripiprazole in major depression. ${ }^{10}$

\section{Brexpiprazole May Have Similar Efficacy to Aripiprazole in Schizophrenia and Major Depression}

Citrome's analysis of efficacy using the number needed to treat (NNT) showed that this was similar for both brexpiprazole and aripiprazole. ${ }^{10}$ Ultimately, the clinical differences in efficacy between these 2 drugs will more likely be seen in individual patients rather than in large multicenter trials, but we will need more clinical experience with brexpiprazole to learn about that.

\section{Summary}

Brexpiprazole and aripiprazole are both dopamine multifunctional agents that act as partial agonists at dopamine 


\section{BRAINSTORMS_Clinical Neuroscience Update}

and serotonin receptors. Both are approved as monotherapies for the treatment of schizophrenia, and as adjunctive treatments to antidepressants for patients with major depressive disorder. Brexpiprazole has different pharmacologic properties than aripiprazole that may predict similar efficacy but improved tolerability, especially for akathisia, EPS, and activation.

\section{References}

1. Stahl SM. Stahl's Essential Psychopharmacology. 4th ed. Cambridge, UK: Cambridge University Press; 2013.

2. Mitzenberg MJ, Yoon JH. An index of relative central $\alpha$-adrenergic receptor antagonism by antipsychotic medications. Exp Clin Psychopharmacol. 2011; 19(1): 31-39.

3. Marek GJ, Aghahanian GK. 5-HT2A receptor or alpha1-adrenoceptor activation induces excitatory postsynaptic currents in layer $\mathrm{V}$ pyramidal cells of the medial prefrontal cortex. Eur J Pharmacol. 1999; 367(2-3): 197-206.

4. Marek GJ, Aghajanian GK. Alpha 1B-adrenoceptor-mediated excitation of piriform cortical interneurons. Eur J Pharmacol. 1996; 305(1-3): 95-100.
5. Pudovkina OL, Cremers T, Westerink B. Regulation of the release of serotonin in the dorsal raphe nucleus by alpha1 and alpha2 adrenoceptors. Synapse. 2003; 50(1): 77-82.

6. Amargós -Bosch M, Adell A, Bortolozzi A, Artigas F. Stimulation of alpha1-adrenoceptors in the rat medial prefrontal cortex increases the local in vivo 5-hydroxytryptamine release: reversal by antipsychotic drugs. J Neurochem. 2003; 87(4): 831-842.

7. Mitrano D, Schroeder JP, Smith Y, et al. $\alpha 1$ adrenergic receptors are localized on presynaptic elements in the nucleus accumbens and regulate mesolimbic dopamine transmission. Neuropsychopharmacology. 2012; 37(9): 2161-2172.

8. Coteccia S. The $\alpha 1$-adrenergic receptors: diversity of signaling networks and regulation. J Recept Signal Transduct Res. 2010; 30(6): 410-419.

9. Stahl SM. Role of $\alpha 1$ adrenergic antagonism in the mechanism of action of iloperidone: reducing extrapyramidal side effects. CNS Spectr. 2013; 18(6): 285-288.

10. Citrome L. The ABC's of dopamine receptor partial agonistsaripiprazole, brexpiprazole and cariprazine: the 15-min challenge to sort these agents out. Int J Clin Pract. 2015; 69(11): 1211-1220. 